\title{
Continuum and Lattice Overlap for Chiral Fermions on the Torus.
}

\author{
C.D. Fosco \\ International Centre for Theoretical Physics \\ P.O. Box 586, 34100 Trieste, Italy
}

October 23, 2018

\begin{abstract}
The overlap formulation is applied to calculate the chiral determinant on a two-dimensional torus with twisted boundary conditions. We evaluate first the continuum overlap, which is convergent and welldefined, and yields the correct string theory result for both the real and imaginary parts of the effective action. We then show that the lattice version of the overlap gives the continuum overlap results in the limit when the lattice spacing tends to zero, and that the subleading terms in that limit are irrelevant.
\end{abstract}




\section{Introduction}

The overlap formalism [1] is a proposal, based on an earlier idea of Kaplan [2], to define fermionic chiral determinants. When implemented on the lattice, it seems to overcome the kinematical constraint stated by the Nielsen-Ninomiya theorem [3], and thus could provide a suitable framework to study nonperturbative phenomena in models containing chiral fermions.

In this method, the determinant of a chiral Dirac operator in $2 d$ dimensions is defined as an overlap between the Dirac vacuum states of two auxiliary Hamiltonians acting on Dirac fermions in $2 d+1$ dimensions.

Different studies and tests have been performed on this new formulation of the problem of evaluating chiral determinants, both in its continuum and lattice versions. In a Hamiltonian approach some tests for the continuum version have been performed in 2 and 4 dimensions in references [4] and [5], respectively, showing that the continuum overlap correctly reproduces some of the properties one expects for a chiral determinant to have, namely chiral anomalies, zero modes, etc. The 4-dimensional continuum results have also been confirmed in a 5-dimensional approach in [7]. Recently, the relation between the phase of the continuum overlap and the $\eta$-invariant has been

elucidated [8]. Regarding the lattice overlap, the main concern has been to show that it does not suffer from any of the drawbacks that afflicted previous attempts to regulate fermions on the lattice. Analytic lattice calculations have been carried out for slowly varying background gauge fields [9, 13, 6]. There are also numerical calculations which confirm the overlap picture $[1$, $11]$.

In this paper we present an expansion of a previous note [1] on the case of fermions with twisted boundary conditions on a torus. Our exact results reinforce the conclusions of [10] where this problem was studied numerically. We consider here both the continuum and lattice version of the overlap for twisted chiral fermions on the torus. We show that the continuum overlap is already regularized and yields a finite answer, that agrees with the one of more standard calculations [12]. The lattice regularization is shown to lead to the continuum overlap in the continuum limit. We also show that the subleading contributions to the continuum calculation vanish when the lattice spacing tends to 0 .

This paper is organized as follows: In section 2 some conventions and definitions used in the formulation of the problem are presented. The contin- 
uum overlap is dealt with in section 3, where the real and imaginary parts of the effective action of the chiral Dirac operator are calculated separately. In section 4 the lattice overlap is considered, showing that it yields the proper continuum limit for both its real and imaginary parts. The subleading terms in the small lattice spacing expansion are shown to be harmless. 


\section{Definitions and conventions.}

We summarize here some technicalities about the Dirac equation and its twisted solutions on a torus with a flat metric. The two-dimensional torus is coordinatized by two real variables

$$
\sigma^{1}, \quad \sigma^{2} \quad, \quad 0 \leq \sigma^{\mu} \leq 1
$$

and is equipped with the Euclidean metric

$$
\begin{aligned}
d s^{2} & =\left|d \sigma^{1}+\tau d \sigma^{2}\right|^{2}=g_{\mu \nu} d \sigma^{\mu} d \sigma^{\nu} \\
g_{\mu \nu} & =\left(\begin{array}{cc}
1 & \tau_{1} \\
\tau_{1} & |\tau|^{2}
\end{array}\right) \quad g^{\mu \nu}=\frac{1}{\tau_{2}^{2}}\left(\begin{array}{cc}
|\tau|^{2} & -\tau_{1} \\
-\tau_{1} & 1
\end{array}\right)
\end{aligned}
$$

where $\tau=\tau_{1}+i \tau_{2}$ and $\tau_{2}>0$. The associated zweibeins $e_{a}^{\mu}$ then follow:

$$
\begin{aligned}
g_{\mu \nu} e_{a}^{\mu} e_{b}^{\nu}= & \delta_{a b}, \\
e_{1}^{\mu}=(1,0) & e_{2}^{\mu}=\left(-\frac{\tau_{1}}{\tau_{2}}, \frac{1}{\tau_{2}}\right) .
\end{aligned}
$$

To define the overlap, we need the vectorlike Dirac operator

$$
\not D=\sigma^{a} e_{a}^{\mu}\left(\partial_{\mu}+i A_{\mu}\right)
$$

where the $\sigma^{a}$ 's are the usual Pauli matrices

$$
\sigma^{1}=\left(\begin{array}{ll}
0 & 1 \\
1 & 0
\end{array}\right) \quad, \quad \sigma^{2}=\left(\begin{array}{cc}
0 & -i \\
i & 0
\end{array}\right) \quad, \quad \sigma^{3}=\left(\begin{array}{cc}
1 & 0 \\
0 & -1
\end{array}\right) \text {. }
$$

We also define Dirac matrices consistent with the metric (2), by the equations

$$
\gamma^{\mu}=\sigma^{a} e_{a}^{\mu}, \quad \mu=1,2 ; \quad \gamma_{5}=\sigma^{3} .
$$

From (3) and (6), some relations follow:

$$
\left\{\gamma^{\mu}, \gamma^{\nu}\right\}=2 g^{\mu \nu},\left\{\gamma^{\mu}, \gamma_{5}\right\}=0, \operatorname{tr}\left(\gamma^{\mu} \gamma^{\nu} \gamma_{5}\right)=\frac{2 i}{\tau_{2}} \epsilon^{\mu \nu}
$$

where $\epsilon^{\mu \nu}$ is the Levi-Civita symbol. Note that $\tau_{2}$ is the volume of the torus. 
We would like to describe twisted fermions on the torus, i.e., the fermionic field has the boundary conditions

$$
\begin{aligned}
& \Psi\left(\sigma^{1}+1, \sigma^{2}\right)=-e^{2 \pi i \varphi_{1}} \Psi\left(\sigma^{1}, \sigma^{2}\right) \\
& \Psi\left(\sigma^{1}, \sigma^{2}+1\right)=-e^{2 \pi i \varphi_{2}} \Psi\left(\sigma^{1}, \sigma^{2}\right),
\end{aligned}
$$

where $\varphi_{1}$ and $\varphi_{2}$ are real numbers such that $0 \leq \varphi_{\mu} \leq 1$. The 'reference' boundary condition about which we define the twistings is antiperiodicity in both directions, hence the minus signs on the rhs in (8). This is to assure that the 'untwisted' case $\left(\varphi_{\mu}=0\right)$, which will be used to normalize the twisted determinant, does not have a zero mode.

To consider free fermions with the twistings (8) is equivalent (by a gauge transformation) to having fermions with antiperiodic boundary conditions in the background of a constant gauge field $A_{\mu}=2 \pi \varphi_{\mu}$, which is the description we shall adopt. Two one-body Dirac Hamiltonians $H_{ \pm}(\varphi)$ in a constant gauge field $A_{\mu}=2 \pi \varphi_{\mu}$ are then defined by

$$
H_{ \pm}(\varphi)=\gamma_{5}(\not \partial+2 \pi i \phi \pm|\Lambda|)
$$

where $\Lambda$ is a constant with the dimensions of a mass.

Translational invariance of both the free and the constant- $A_{\mu}$ cases suggests the use of Fourier transforms. The Fourier transform of an antiperiodic function $f$, depending on the coordinates $\sigma^{\mu}$ will be given by

$$
f(\sigma)=\sum_{n} e^{i 2 \pi n_{\mu} \sigma^{\mu}} \tilde{f}(n), \quad \tilde{f}(n)=\int d^{2} \sigma e^{-i 2 \pi n_{\mu} \sigma^{\mu}} f(\sigma)
$$

where $n^{\mu}$ runs over all the half-integers.

The free $(\varphi=0)$, positive $\left(u_{ \pm}\right)$, and negative $\left(v_{ \pm}\right)$eigenspinors in Fourier space satisfy

$$
\tilde{H}_{ \pm}(n) u_{ \pm}(n)=\omega(n) u_{ \pm}(n) ; \quad \tilde{H}_{ \pm}(n) v_{ \pm}(n)=-\omega(n) v_{ \pm}(n)
$$

where

$$
\begin{array}{ccc}
\tilde{H}_{ \pm}(n)=\gamma_{5}(2 \pi i \not h \pm|\Lambda|) & \omega(n)=\sqrt{(2 \pi)^{2} n^{2}+\Lambda^{2}} \\
u_{ \pm}(n)=\frac{\omega(n) \pm|\Lambda|-2 \pi i \not h}{\sqrt{2 \omega(n)(\omega(n) \pm|\Lambda|)}} \chi & v_{ \pm}(n)=\frac{\omega(n) \mp|\Lambda|+2 \pi i \not h}{\sqrt{2 \omega(n)(\omega(n) \mp|\Lambda|)}} \chi
\end{array}
$$


and $\chi=\left(\begin{array}{l}1 \\ 0\end{array}\right)$.

It should be evident from (8) that the eigenspinors of $\tilde{H}(n \mid \varphi)$, the Fourier transform of $H_{ \pm}(\varphi)$, are equal to those of $H_{ \pm}(0)$ but with shifted $n$ 's: $n \rightarrow n+\varphi$,

$$
\begin{aligned}
\tilde{H}_{ \pm}(n \mid \varphi) u_{ \pm}(n \mid \varphi) & =\omega(n \mid \varphi) u_{ \pm}(n \mid \varphi) \\
\tilde{H}_{ \pm}(n \mid \varphi) v_{ \pm}(n \mid \varphi) & =-\omega(n \mid \varphi) v_{ \pm}(n \mid \varphi) \\
u_{ \pm}(n \mid \varphi)=u_{ \pm}(n+\varphi) & , \quad v_{ \pm}(n \mid \varphi)=v_{ \pm}(n+\varphi),
\end{aligned}
$$

where $\omega(n \mid \varphi)=\omega(n+\varphi)$.

\section{Continuum overlap and effective action.}

Using the set of eigenspinors introduced in (13), we expand the fermionic fields

$$
\Psi(\sigma)=\sum_{n}\left(b_{ \pm}(n \mid \varphi) u_{ \pm}(n \mid \varphi)+d_{ \pm}^{\dagger}(n \mid \varphi) v_{ \pm}(n \mid \varphi)\right) e^{i 2 \pi n \cdot \sigma}
$$

which contains the free $(\varphi=0)$ field expansion as a particular case. The operators involved in (14) satisfy the anticommutation relations

$$
\begin{aligned}
\left\{\Psi(\sigma), \Psi\left(\sigma^{\prime}\right)\right\}=0 & \left\{\Psi(\sigma), \Psi^{\dagger}\left(\sigma^{\prime}\right)\right\}=\delta\left(\sigma-\sigma^{\prime}\right) \\
\left\{b_{ \pm}(n \mid \varphi), b_{ \pm}^{\dagger}\left(n^{\prime} \mid \varphi\right)\right\}=\delta_{n, n^{\prime}} & \left\{d_{ \pm}(n \mid \varphi), d_{ \pm}^{\dagger}\left(n^{\prime} \mid \varphi\right)\right\}=\delta_{n, n^{\prime}} \\
\left\{b_{ \pm}(n \mid \varphi), b_{ \pm}\left(n^{\prime} \mid \varphi\right)\right\}=0 & \left\{d_{ \pm}(k \mid \varphi), d_{ \pm}\left(n^{\prime} \mid \varphi\right)\right\}=0,
\end{aligned}
$$

where $\delta\left(\sigma-\sigma^{\prime}\right)$ is periodic in $\sigma$, with period equal to 1 . With these conventions, the corresponding Dirac vacua, i.e. the vectors obtained by filling the negative energy states, are defined by

$$
|\varphi \pm\rangle=\prod_{n} d_{ \pm}(n \mid \varphi)|0\rangle \quad|0 \pm\rangle=\prod_{n} d_{ \pm}(n \mid 0)|0\rangle
$$

and the overlap definition of the normalized determinant becomes

$$
\frac{\operatorname{det} D(\varphi)}{\operatorname{det} D(0)}=\lim _{\Lambda \rightarrow \infty}\left[\frac{\langle+\mid \varphi+\rangle}{|\langle+\mid \varphi+\rangle|} \frac{\langle\varphi+\mid \varphi-\rangle}{\langle+\mid-\rangle} \frac{\langle\varphi-\mid-\rangle}{|\langle\varphi-\mid-\rangle|}\right]
$$


where $D(\varphi)$ denotes the chiral Dirac operator: $D(\varphi)=(\not \partial+2 \pi i \not) \frac{\left(1+\gamma_{5}\right)}{2}$.

From (17) we also define the Euclidean effective action

$$
\Gamma(\varphi)=-\log \left[\frac{\operatorname{det} D(\varphi)}{\operatorname{det} D(0)}\right] .
$$

Equations (17) and (18) can be written in terms of the eigenspinors introduced in section 2. For example,

$$
\begin{aligned}
& \Gamma(\varphi, \Lambda)= \\
& \quad-\sum_{n} \log \left(\frac{v_{+}^{\dagger}(n) v_{+}(n+\varphi)}{\left|v_{+}^{\dagger}(n) v_{+}(n+\varphi)\right|} \frac{v_{+}^{\dagger}(n+\varphi) v_{-}(n+\varphi)}{v_{+}^{\dagger}(n) v_{-}(n)} \frac{v_{-}^{\dagger}(n+\varphi) v_{-}(n)}{\left|v_{-}^{\dagger}(n+\varphi) v_{-}(n)\right|}\right)
\end{aligned}
$$

where we have indicated the $\Lambda$-dependence of $\Gamma$ explicitly, to make it clear that the series on the rhs of (19) shall be evaluated first, taking the $\Lambda \rightarrow \infty$ limit afterwards:

$$
\Gamma(\varphi)=\lim _{\Lambda \rightarrow \infty} \Gamma(\varphi, \Lambda) .
$$

From (17), (18) and (19), with the particular phase conventions chosen for the eigenspinors, the real and imaginary parts of $\Gamma(\varphi, \Lambda)$ are

$$
\begin{aligned}
\operatorname{Re} \Gamma(\varphi, \Lambda) & =-\log \left(\frac{\langle\varphi+\mid \varphi-\rangle}{\langle+\mid-\rangle}\right)=-\sum_{n} \log \left(\frac{v_{+}^{\dagger}(n+\varphi) v_{-}(n+\varphi)}{v_{+}^{\dagger}(n) v_{-}(n)}\right) \\
\operatorname{Im} \Gamma(\varphi, \Lambda) & =-\operatorname{Im} \log \left(\frac{\langle+\mid \varphi+\rangle}{|\langle+\mid \varphi+\rangle|} \frac{|\langle-\mid \varphi-\rangle|}{\langle-\mid \varphi-\rangle}\right) \\
& =-\operatorname{Im} \sum_{n} \log \left(\frac{v_{+}^{\dagger}(n) v_{+}(n+\varphi)}{\left|v_{+}^{\dagger}(n) v_{+}(n+\varphi)\right|} \frac{\left|v_{-}^{\dagger}(n) v_{-}(n+\varphi)\right|}{v_{-}^{\dagger}(n) v_{-}(n+\varphi)}\right) .(21)
\end{aligned}
$$

We shall deal with the real and imaginary parts of the effective action separatedly in the following subsections. 


\subsection{Real part of the effective action.}

Using the explicit form for the eigenspinors ( (12) and (13)) in (21), we obtain for $\operatorname{Re} \Gamma(\varphi, \Lambda)$ the following double-series representation:

$\operatorname{Re} \Gamma(\varphi, \Lambda)=\frac{1}{2} \sum_{n} \log \left(1+\frac{\Lambda^{2}}{(2 \pi)^{2}(n+\varphi)^{2}}\right)-\frac{1}{2} \sum_{n} \log \left(1+\frac{\Lambda^{2}}{(2 \pi)^{2} n^{2}}\right)$.

With the explicit form of the metric tensor introduced in (2), and defining

the parameter $\lambda=\frac{\tau_{2}|\Lambda|}{2 \pi}$, we can rewrite the real part in a more expanded form as

$\operatorname{Re} \Gamma(\varphi, \Lambda)=$

$$
\begin{gathered}
\frac{1}{2} \sum_{n_{1}, n_{2}} \log \left(\frac{|\tau|^{2}\left(n_{1}+\varphi_{1}\right)^{2}-2 \tau_{1}\left(n_{1}+\varphi_{1}\right)\left(n_{2}+\varphi_{2}\right)+\left(n_{2}+\varphi_{2}\right)^{2}+\lambda^{2}}{|\tau|^{2}\left(n_{1}+\varphi_{1}\right)^{2}-2 \tau_{1}\left(n_{1}+\varphi_{1}\right)\left(n_{2}+\varphi_{2}\right)+\left(n_{2}+\varphi_{2}\right)^{2}}\right) \\
-\frac{1}{2} \sum_{n_{1}, n_{2}} \log \left(\frac{|\tau|^{2} n_{1}^{2}-2 \tau_{1} n_{1} n_{2}+n_{2}^{2}+\lambda^{2}}{|\tau|^{2} n_{1}^{2}-2 \tau_{1} n_{1} n_{2}+n_{2}^{2}}\right) .
\end{gathered}
$$

Direct evaluation of the double series in (23) is not a straightforward task as it stands, but we can simplify the calculation by taking derivatives with respect to $\lambda$ in both sides of (23), obtaining the differential equation

$$
\begin{aligned}
\frac{\partial}{\partial \lambda} \operatorname{Re}(\varphi, \lambda) & =\lambda\left[\sum_{n_{1}, n_{2}}\left(\left|\tau\left(n_{1}+\varphi_{1}\right)-\left(n_{2}+\varphi_{2}\right)\right|^{2}+\lambda^{2}\right)^{-1}\right. \\
& \left.-\sum_{n_{1}, n_{2}}\left(\left|\tau n_{1}-n_{2}\right|^{2}+\lambda^{2}\right)^{-1}\right]
\end{aligned}
$$

where now the rhs of (24) can indeed be evaluated by standard methods. Let us first note that this expression is convergent, as may for example be verified by expanding the first series in powers of $\varphi_{\mu}$. The term of order zero which might produce a logarithmic divergence in the first series is exactly cancelled by the second series, and the successive terms produce series that behave as

\footnotetext{
${ }^{1}$ In our normalization $0 \leq \varphi_{\mu}<1$. Arguments based in series expansions in powers of $\varphi_{\mu}$ will be used frequently in the following.
} 
$|n|^{-4}$ at worst, for large $|n|$ (in fact, the term of order $q$ in $\varphi$ behaves like $|n|^{-2-q}$, and only even $q$ 's appear), thus they converge.

To evaluate the rhs of (24) we shall first perform the summation over one of the half-integer indices, $n_{2}$, say. The kind of expression one needs to evaluate here will also appear in dealing with the imaginary part of $\Gamma$. It has the following structure

$$
f(x, y)=\sum_{n_{2}}\left[\left(n_{2}-x\right)^{2}+y^{2}\right]^{-1}
$$

where $x$ and $y$ are functions of $n_{1}$ as well as of $\varphi_{\mu}$ and $\lambda$. Then we resort to the procedure, familiar in finite temperature quantum field theory, of converting the series (25) into an integral along a path in the complex plane, by introducing a function which has poles at the half-integer real numbers:

$$
f(x, y)=\oint_{C} \frac{d z}{2 \pi i} \pi \tan (\pi z)(z-x+i y)^{-1}(z-x-i y)^{-1}
$$

where $C$ is a path that encircles all the poles of the tan counterclockwise f? while avoiding the poles at $z=x \pm i y$. By deforming $C$, we can evaluate the integral by knowing the residues at the poles $z_{ \pm}=x \pm i y$. It yields

$$
f(x, y)=\frac{\pi}{2 i y}[\tan (\pi(x+i y))-\tan (\pi(x-i y))] .
$$

Applying the result (27) to (24), we get

$$
\begin{aligned}
& \frac{\partial}{\partial \lambda} \operatorname{Re} \Gamma(\varphi, \lambda)= \\
& \frac{\pi \lambda}{2 i} \times \sum_{n_{1}}\left\{\frac { 1 } { \eta ( \varphi ) } \left[\tan \left(\pi\left(\varphi_{2}+\tau_{1}\left(n_{1}+\varphi_{1}\right)+i \eta(\varphi)\right)\right)\right.\right. \\
&\left.\quad-\tan \left(\pi\left(\varphi_{2}+\tau_{1}\left(n_{1}+\varphi_{1}\right)-i \eta(\varphi)\right)\right)\right] \\
&\left.-\frac{1}{\eta(0)}\left[\tan \left(\pi\left(\tau_{1} n_{1}+i \eta(0)\right)\right)+\tan \left(\pi\left(\tau_{1} n_{1}-i \eta(0)\right)\right)\right]\right\},
\end{aligned}
$$

\footnotetext{
${ }^{2}$ This procedure is valid provided $y>0$, which is true for any $\Lambda \neq 0$.
} 
where $\eta(\varphi)=\sqrt{\tau_{2}^{2}\left(n_{1}+\varphi_{1}\right)^{2}+\lambda^{2}}$. The next step is to integrate the differential equation (28), using the boundary condition $\operatorname{Re} \Gamma(\varphi, 0)=0$, which follows from (23). This integration is elementary, the answer being

$$
\begin{aligned}
\operatorname{Re} \Gamma(\varphi, \lambda) & =\frac{1}{2} \sum_{n_{1}}\left\{\log \frac{\cos \left[\pi\left(\varphi_{2}+\tau_{1}\left(n_{1}+\varphi_{1}\right)+i \sqrt{\lambda^{2}+\tau_{2}^{2}\left(n_{1}+\varphi_{1}\right)^{2}}\right)\right]}{\cos \left[\pi\left(\tau_{1} n_{1}+i \sqrt{\lambda^{2}+\tau_{2}^{2} n_{1}^{2}}\right)\right]}\right. \\
& +\log \frac{\cos \left[\pi\left(\varphi_{2}+\tau_{1}\left(n_{1}+\varphi_{1}\right)-i \sqrt{\lambda^{2}+\tau_{2}^{2}\left(n_{1}+\varphi_{1}\right)^{2}}\right)\right]}{\cos \left[\pi\left(\tau_{1} n_{1}-i \sqrt{\lambda^{2}+\tau_{2}^{2} n_{1}^{2}}\right)\right]} \\
& -\log \frac{\cos \left[\pi\left(\varphi_{2}+\tau_{1}\left(n_{1}+\varphi_{1}\right)+i \tau_{2}\left|n_{1}+\varphi_{1}\right|\right)\right]}{\cos \left[\pi\left(\tau_{1} n_{1}+i \tau_{2}\left|n_{1}\right|\right)\right]} \\
& \left.-\log \frac{\cos \left[\pi\left(\varphi_{2}+\tau_{1}\left(n_{1}+\varphi_{1}\right)-i \tau_{2}\left|n_{1}+\varphi_{1}\right|\right)\right]}{\cos \left[\pi\left(\tau_{1} n_{1}-i \tau_{2}\left|n_{1}\right|\right)\right]}\right\}
\end{aligned}
$$

So far no approximation has been made in deriving (29), which is exact for any $\lambda(\propto|\Lambda|)$. To obtain $\operatorname{Re} \Gamma(\varphi)$ we shall take the $\lambda \rightarrow \infty$ limit. Observing that (29) may be decomposed into a $\lambda$-independent plus a $\lambda$-dependent piece, we will find a more explicit expression for the former first, and then take the limit $\lambda \rightarrow \infty$ for the latter

$$
\begin{aligned}
{[\operatorname{Re} \Gamma(\varphi, \lambda)]_{\lambda-\text { indep }}=} & \\
& -\frac{1}{2} \sum_{n_{1}} \log \frac{\cos \left[\pi\left(\varphi_{2}+\tau_{1}\left(n_{1}+\varphi_{1}\right)+i \tau_{2}\left|n_{1}+\varphi_{1}\right|\right)\right]}{\cos \left[\pi\left(\tau_{1} n_{1}+i \tau_{2}\left|n_{1}\right|\right)\right]} \\
& -\frac{1}{2} \sum_{n_{1}} \log \frac{\cos \left[\pi\left(\varphi_{2}+\tau_{1}\left(n_{1}+\varphi_{1}\right)-i \tau_{2}\left|n_{1}+\varphi_{1}\right|\right)\right]}{\cos \left[\pi\left(\tau_{1} n_{1}-i \tau_{2}\left|n_{1}\right|\right)\right]} .
\end{aligned}
$$

Using the relation

$$
\sum_{n} \log \frac{\cos [\pi(\tau n+\alpha)]}{\cos (\pi \tau n)}=\log \frac{\vartheta(\alpha, \tau)}{\vartheta(0, \tau)}
$$

where $n$ is half-integer and $\vartheta$ is a $\vartheta$-function (whose infinite-product representation implies (31)) which can be defined by the (single) series

$$
\vartheta(\alpha, \tau)=\sum_{n} e^{i \pi \tau n^{2}+2 \pi i n \alpha},
$$


one can verify after a bit of algebra that

$$
[\operatorname{Re} \Gamma(\varphi, \lambda)]_{\lambda-\text { indep }}=-\operatorname{Re} \log \frac{\vartheta(\alpha, \tau)}{\vartheta(0, \tau)}, \alpha=\tau \varphi_{1}-\varphi_{2}
$$

Now we have to take the $\lambda \rightarrow \infty$ limit [3 of the remaining, $\lambda$-dependent part

$$
\begin{aligned}
& {[\operatorname{Re} \Gamma(\varphi, \lambda)]_{\lambda-\text { dep }}=} \\
& \quad \frac{1}{2} \sum_{n_{1}}\left\{\log \frac{\cos \left[\pi\left(\varphi_{2}+\tau_{1}\left(n_{1}+\varphi_{1}\right)+i \sqrt{\lambda^{2}+\tau_{2}^{2}\left(n_{1}+\varphi_{1}\right)^{2}}\right)\right]}{\cos \left[\pi\left(\tau_{1} n_{1}+i \sqrt{\lambda^{2}+\tau_{2}^{2} n_{1}^{2}}\right)\right]}\right. \\
& \left.\quad+\log \frac{\cos \left[\pi\left(\varphi_{2}+\tau_{1}\left(n_{1}+\varphi_{1}\right)-i \sqrt{\lambda^{2}+\tau_{2}^{2}\left(n_{1}+\varphi_{1}\right)^{2}}\right)\right]}{\cos \left[\pi\left(\tau_{1} n_{1}-i \sqrt{\lambda^{2}+\tau_{2}^{2} n_{1}^{2}}\right)\right]}\right\} .
\end{aligned}
$$

The $|\lambda| \rightarrow \infty$ limit of (34) is difficult to calculate as it stands, because of the non-analytic behaviour in $\frac{1}{\lambda}$. However, writing the cos in (34) in terms of exponentials, and keeping the leading terms (behaving as $e^{|\lambda|}$ ), one can prove that only terms of up to order 2 in $\varphi$ yield non-vanishing contributions when $\lambda \rightarrow \infty$ :

$$
[\operatorname{Re} \Gamma(\varphi, \lambda)]_{\lambda-d e p}=\pi \sum_{n_{1}}\left[0+\frac{1}{2} \frac{\tau_{2}^{2} \lambda^{2}}{\left(\lambda^{2}+\tau_{2}^{2} n_{1}^{2}\right)^{\frac{3}{2}}} \varphi_{1}^{2}+\mathcal{O}\left(\varphi^{4}\right)\right] .
$$

The $\varphi$-independent term vanishes identically, whereas the odd powers are absent by symmetry. All the higher-order terms not shown in (35) are given by convergent series and vanish in the limit $\lambda \rightarrow \infty$. Thus

$$
\lim _{\lambda \rightarrow \infty}[\operatorname{Re} \Gamma(\varphi, \lambda)]_{\lambda-d e p}=\varphi_{1}^{2} \lim _{\lambda \rightarrow \infty} \frac{\pi}{2} \sum_{n_{1}} \frac{\tau_{2}^{2} \lambda^{2}}{\left(\lambda^{2}+\tau_{2}^{2} n_{1}^{2}\right)^{\frac{3}{2}}}
$$

which can be calculated by replacing the series by an integral, since the error of this replacement is made arbitrarily small by just increasing $\lambda$. Then

$$
\lim _{\lambda \rightarrow \infty}[\operatorname{Re} \Gamma(\varphi, \lambda)]_{\lambda-d e p}=\varphi_{1}^{2} \lim _{\lambda \rightarrow \infty} \frac{\pi}{2} \int_{-\infty}^{+\infty} d x \frac{\tau_{2}^{2} \lambda^{2}}{\left(\lambda^{2}+\tau_{2}^{2} x^{2}\right)^{\frac{3}{2}}}=\pi \tau_{2} \varphi_{1}^{2} .
$$

\footnotetext{
${ }^{3}$ If dimensions were introduced, this limit would correspond to the ratio between the momentum spacing and $\Lambda$ going to zero.
} 
Putting together the results for the $\lambda$-independent and $\lambda$-dependent parts, we obtain the final result for the real part of the effective action:

$$
\operatorname{Re} \Gamma(\varphi, \lambda)=-\operatorname{Re} \log \left(\frac{\vartheta(\alpha, \tau)}{\vartheta(0, \tau)}\right)+\pi \tau_{2} \varphi_{1}^{2}
$$

\subsection{Imaginary part of the effective action.}

In the continuum overlap definition of the imaginary part (21), we make use again of the explicit form of the eigenspinors to obtain

$$
\operatorname{Im} \Gamma(\varphi, \Lambda)=\operatorname{Im} Z(\varphi, \Lambda)
$$

where

$$
\begin{aligned}
& Z(\varphi, \Lambda)= \\
& \sum_{n} \log \left[(\omega(n)+|\Lambda|)(\omega(n+\varphi)+|\Lambda|)+(2 \pi)^{2} n \cdot(n+\varphi)+\frac{(2 \pi)^{2} i}{\tau_{2}} \epsilon^{\mu \nu} n_{\mu} \varphi_{\nu}\right] \\
& -\sum_{n} \log \left[(\omega(n)-|\Lambda|)(\omega(n+\varphi)-|\Lambda|)+(2 \pi)^{2} n \cdot(n+\varphi)+\frac{(2 \pi)^{2} i}{\tau_{2}} \epsilon^{\mu \nu} n_{\mu} \varphi_{\nu}\right] .
\end{aligned}
$$

We now proceed to evaluate $Z(\varphi, \lambda)$, keeping in mind that its real part is irrelevant to the calculation of the imaginary part of $\Gamma$, and we shall often ignore real terms in $Z$. This implies that we shall not be concerned with the convergence of $Z$ in (40) (which looks badly divergent), but rather with the convergence of its imaginary part only. Again, it is not clear how to evaluate the series defining $Z$ as it stands. However, a procedure similar to the one used in the calculation of the real part can be applied, if one first simplifies expression (40). We cannot sum exactly the series for an arbitrary $\Lambda$, as it was the case for the real part, due to the presence of square roots. A simplification is achieved by taking the $\Lambda \rightarrow \infty$ limit before summing over $n$. We are entitled to interchange the order of summation and limit because the imaginary part of $Z$ is convergent for any $\Lambda$, even for $\Lambda \rightarrow \infty$. To show this, we first write the imaginary part of $Z$ more explicitly: 
$\operatorname{Im} Z(\varphi, \Lambda)$

$$
\begin{gathered}
=\sum_{n} \arctan \left[\frac{(2 \pi)^{2}}{\tau_{2}} \frac{\epsilon^{\mu \nu} n_{\mu} \varphi_{\nu}}{(\omega(n)+\Lambda)(\omega(n+\varphi)+\Lambda)+(2 \pi)^{2} n \cdot(n+\varphi)}\right] \\
-\sum_{n} \arctan \left[\frac{(2 \pi)^{2}}{\tau_{2}} \frac{\epsilon^{\mu \nu} n_{\mu} \varphi_{\nu}}{(\omega(n)-\Lambda)(\omega(n+\varphi)-\Lambda)+(2 \pi)^{2} n \cdot(n+\varphi)}\right] .
\end{gathered}
$$

To study the convergence of each one of the series in (41) (corresponding to the two possible signs of $\Lambda$ ), we analyze the behaviour of the corresponding summands when $n \rightarrow \infty$. For sufficiently large $n$, each one of the expressions between brackets in (41) behaves at worst like $|n|^{-1}$, which can of course be made much smaller than one. Thus in analyzing the convergence of the series we can replace the arctan's by their small-arguments, i.e.,

$$
\begin{aligned}
& \arctan {\left[\frac{(2 \pi)^{2}}{\tau_{2}} \frac{\epsilon^{\mu \nu} n_{\mu} \varphi_{\nu}}{(\omega(n)+\Lambda)(\omega(n+\varphi)+\Lambda)+(2 \pi)^{2} n \cdot(n+\varphi)}\right] } \\
& \simeq \quad \frac{(2 \pi)^{2}}{\tau_{2}} \frac{\epsilon^{\mu \nu} n_{\mu} \varphi_{\nu}}{(\omega(n)+\Lambda)(\omega(n+\varphi)+\Lambda)+(2 \pi)^{2} n \cdot(n+\varphi)}
\end{aligned}
$$

and analogously for the second term in (41). Now we study the convergence of (the sum over $n$ of) (42). Again we follow the approach of performing an expansion in powers of $\varphi$, and showing that the terms which might produce divergences actually vanish when summed over $n$. It is not difficult to realize that only even powers of $\varphi_{\mu}$ are allowed by symmetry, and that terms with 4 powers or more of $\varphi$ are convergent. Thus we only need to prove the convergence of the second-order term

$$
\begin{aligned}
\sum_{n} \frac{(2 \pi)^{2}}{\tau_{2}} & \frac{\epsilon^{\mu \nu} n_{\mu} \varphi_{\nu}}{(\omega(n)+\Lambda)(\omega(n+\varphi)+\Lambda)+(2 \pi)^{2} n \cdot(n+\varphi)} \\
= & \frac{(2 \pi)^{4}}{\tau_{2}} \epsilon^{\mu \nu} \varphi_{\nu} \varphi^{\lambda} \sum_{n} \frac{2+\frac{\Lambda}{\omega(n)}}{(\omega(n)+\Lambda)^{4}} n_{\mu} n_{\lambda}+\mathcal{O}\left(\varphi^{4}\right) .
\end{aligned}
$$

The quadratic term in (43) vanishes by symmetry, because

$$
\sum_{n} \frac{2+\frac{\Lambda}{\omega(n)}}{(\omega(n)+\Lambda)^{4}} n_{\mu} n_{\lambda} \propto g_{\mu \lambda},
$$


and thus the second order term becomes proportional to

$$
\epsilon^{\mu \nu} \varphi_{\nu} \varphi^{\lambda} g_{\mu \lambda}=\epsilon^{\mu \nu} \varphi_{\mu} \varphi_{\nu}=0 .
$$

Having shown that the series wich defines the imaginary part of the effective action is convergent for any $\Lambda$, we take the limit $\Lambda \rightarrow \infty$ before summing over $n$. In this limit the first log in (40) yields a vanishing contribution to the imaginary part of the effective action, since

$$
\omega(n)+|\Lambda| \rightarrow \mathcal{O}(|\Lambda|)
$$

when $|\Lambda| \rightarrow \infty$ and this suppress the imaginary part of the first log by a power of $|\Lambda|^{-1}$. Regarding the second log in (40), the different sign in front of $|\Lambda|$ produces the asymptotic behaviour

$$
\omega(n)-|\Lambda| \rightarrow \mathcal{O}\left(|\Lambda|^{-1}\right)
$$

Thus when $\Lambda \rightarrow \infty$ the non-vanishing contributions originate only from the second log in (40) and they are given by the imaginary part of f

$$
Z(\varphi, \Lambda)=-\sum_{n} \log \left[n \cdot(n+\varphi)+\frac{i}{\tau_{2}} \epsilon^{\mu \nu} n_{\mu} \varphi_{\nu}\right]
$$

Now we perform the summation over $n$. It is convenient to introduce an auxiliary variable $\epsilon$ into (48), in order to be able to calculate this series:

$$
Z(\varphi)=-\lim _{\epsilon \rightarrow 0} \sum_{n} \log \left[\epsilon^{2}+n \cdot(n+\varphi)+\frac{i}{\tau_{2}} \epsilon^{\mu \nu} n_{\mu} \varphi_{\nu}\right]
$$

The rest of the evaluation becomes akin to the one already performed for the real part of the effective action. We differentiate (49) with respect to $\epsilon$ :

$$
\begin{aligned}
\frac{\partial}{\partial \epsilon} Z(\varphi, \epsilon) & \\
& =-2 \epsilon \sum_{n}\left[\epsilon^{2}+|\tau|^{2} n_{1}\left(n_{1}+\varphi_{1}\right)-\tau_{1} n_{1}\left(n_{2}+\varphi_{2}\right)\right.
\end{aligned}
$$

\footnotetext{
${ }^{4}$ The large momentum behaviour of the terms in the sum defining the imaginary part is much milder than the ones corresponding to the real part. This may be expected from the fact that the imaginary part is related to the chiral anomaly.

${ }^{5}$ We remind the reader that the divergence of $(48)$ is irrelevant for our discussion as we are interested in $\operatorname{Im} Z(\varphi, \Lambda)$, which as argued above, is convergent. Similar arguments will be used again in the next section.
} 


$$
\left.-\tau_{1} n_{2}\left(n_{1}+\varphi_{1}\right)+n_{2}\left(n_{2}+\varphi_{2}\right)+i \tau_{2}\left(n_{1} \varphi_{2}-n_{2} \varphi_{1}\right)\right]^{-1}
$$

and then perform the summation over the index $n_{2}$. The sum we are faced with in (50) is convergent for the same reasons as the corresponding one for the real part. The resulting differential equation has however a different kind of boundary condition, namely $\lim _{\epsilon \rightarrow \infty} \operatorname{Im} Z(\varphi, \epsilon)=0$.

After summing over $n_{2}$ and integrating between two values $\epsilon_{2}, \epsilon_{1}$, we obtain

$$
\begin{aligned}
Z\left(\varphi, \epsilon_{1}\right)-Z\left(\varphi, \epsilon_{2}\right) & =\sum_{n_{1}}\left[-\log \cos \pi\left(\tau_{1} n_{1}+\frac{\alpha}{2}+i s\left(\epsilon_{1}\right)\right)\right. \\
& \left.-\log \cos \pi\left(\tau_{1} n_{1}+\frac{\alpha}{2}-i s\left(\epsilon_{1}\right)\right)\right]-\left(\epsilon_{1} \leftrightarrow \epsilon_{2}\right)
\end{aligned}
$$

where $s(\epsilon)=\sqrt{\epsilon^{2}-\left(\frac{\alpha}{2}+i \tau_{2} n_{1}\right)^{2}}$. Of course we are interested in the $\lim _{\epsilon \rightarrow 0} Z(\varphi, \epsilon)$, so we derive it from (51) by letting $\epsilon_{1}$ go to 0 and $\epsilon_{2}$ to $\infty$ :

$$
\begin{aligned}
Z(\varphi) & =\lim _{\epsilon \rightarrow 0} Z(\varphi, \epsilon)=-\sum_{n_{1}} \log \left[\cos \pi\left(\tau n_{1}+\alpha\right) \cos \pi \bar{\tau} n_{1}\right] \\
& +\lim _{\epsilon_{2} \rightarrow \infty} \sum_{n_{1}}\left[\log \cos \pi\left(\tau_{1} n_{1}+\frac{\alpha}{2}+i \sqrt{\epsilon_{2}^{2}-\left(\frac{\alpha}{2}+i \tau_{2} n_{1}\right)^{2}}\right)\right. \\
& \left.+\log \cos \pi\left(\tau_{1} n_{1}+\frac{\alpha}{2}-i \sqrt{\epsilon_{2}^{2}-\left(\frac{\alpha}{2}+i \tau_{2} n_{1}\right)^{2}}\right)\right] .
\end{aligned}
$$

The contribution of the first sum in (52) to the imaginary part of $Z(\varphi, \Lambda)$ can be rewritten as

$$
\begin{aligned}
-\operatorname{Im} \sum_{n_{1}} \log \left[\cos \pi\left(\tau n_{1}+\alpha\right) \cos \pi \bar{\tau} n_{1}\right] & =-\operatorname{Im} \sum_{n_{1}} \log \left[\frac{\cos \pi\left(\tau n_{1}+\alpha\right)}{\cos \pi \tau n_{1}}\right] \\
& =-\operatorname{Im} \log \left[\frac{\vartheta(\alpha, \tau)}{\vartheta(0, \tau)}\right]
\end{aligned}
$$

This fits exactly with the result for the $\lambda$-independent part in (33), to yield the full $\vartheta$-function dependence of the effective action. The limiting procedure for the second term in (52) is analogous to the one followed in the calculation of (34) 37):

$$
\lim _{\epsilon_{2} \rightarrow \infty} \quad \operatorname{Im} \sum_{n_{1}}\left[\log \cos \pi\left(\tau_{1} n_{1}+\frac{\alpha}{2}+i \sqrt{\epsilon_{2}^{2}-\left(\frac{\alpha}{2}+i \tau_{2} n_{1}\right)^{2}}\right)\right.
$$




$$
\begin{aligned}
& \left.+\log \cos \pi\left(\tau_{1} n_{1}+\frac{\alpha}{2}-i \sqrt{\epsilon_{2}^{2}-\left(\frac{\alpha}{2}+i \tau_{2} n_{1}\right)^{2}}\right)\right] \\
& =2 \pi \operatorname{Im} \lim _{\epsilon_{2} \rightarrow \infty} \sum_{n_{1}}\left[\sqrt{\epsilon_{2}^{2}-\left(\frac{\alpha}{2}+i \tau_{2} n_{1}\right)^{2}}-\sqrt{\epsilon_{2}^{2}+\tau_{2}^{2} n_{1}^{2}}\right] \\
& =-\operatorname{Im} \frac{\pi \alpha^{2}}{2 \tau_{2}} \\
& =-\tau_{1} \pi \varphi_{1}^{2}+\pi \varphi_{1} \varphi_{2} .
\end{aligned}
$$

Substituting (53) and (54) in (52), we get

$$
\operatorname{Im} Z(\varphi)=-\operatorname{Im} \log \frac{\vartheta(\alpha, \tau)}{\vartheta(0, \tau)}-\tau_{1} \pi \varphi_{1}^{2}+\pi \varphi_{1} \varphi_{2}=\operatorname{Im} \Gamma(\varphi)
$$

The combination of this result with (38) leads us to the final form of the effective action

$$
\Gamma(\varphi)=-\log \frac{\vartheta(\alpha, \tau)}{\vartheta(0, \tau)}-i \pi \tau \varphi_{1}^{2}+i \pi \varphi_{1} \varphi_{2} .
$$

This agrees with the string theory evaluation of the determinant of twisted fermions on a 2-torus [9], and with the numerical lattice calculation of ref. [10]. 


\section{Lattice overlap.}

We now consider the problem of calculating the effective action when the system is defined on a lattice of $N^{2}$ sites, which discretize the torus. Although Equation (17) remains formally the same for this case, the Dirac vacua must be constructed from the corresponding lattice eigenspinors instead. The discretized lattice is defined by the set of points $\sigma^{\mu}=a t^{\mu}$, where $t^{\mu}$ are integers such that $0 \leq t^{\mu} \leq N$, and $a$ is the lattice spacing: $a=\frac{1}{N}$, where the last relation follows from the requirement that for the continuum torus $0 \leq \sigma^{\mu} \leq 1$. To the fermionic field operators on the continuum, there correspond the lattice ones $\Psi(\sigma) \rightarrow \Psi(a t)$, and the second-quantized Dirac Hamiltonian is

$$
\hat{H}_{ \pm}(\varphi)=\sum_{t, s} \Psi^{\dagger}(a t) H_{ \pm}(a(t-s)) U(a t, a s) \Psi(a s)
$$

where $H_{ \pm}$is the lattice one-body Hamiltonian, which we define in terms of its Fourier transform

$$
\begin{aligned}
H_{ \pm}(a(r-s)) & =\frac{1}{N^{2}} \sum_{-N / 2}^{+N / 2} \tilde{H}_{ \pm}(n) e^{2 \pi i a n \cdot(r-s)} \\
\tilde{H}_{ \pm}(n) & =\frac{1}{a} \sigma_{3}(i \not(2 \pi a n)+B(2 \pi a n) \pm a|\Lambda|) .
\end{aligned}
$$

The link variables adopt a very simple, and moreover, translation-invariant form

$$
U(a r, a s)=e^{-i \int_{a s}^{a r} d x \cdot A}=e^{-2 \pi i a \varphi \cdot(r-s)},
$$

and the functions $C_{\mu}$ and $B$ are defined by

$$
\begin{aligned}
C_{\mu}(a n) & =\sin \left(a n_{\mu}\right) \\
B(a n) & =r \sum_{\mu=1,2}\left(1-\cos a n_{\mu}\right)
\end{aligned}
$$

where $r$ is a number. Our conventions for the 1-body Hamiltonian are so chosen as to assure that it tends to its continuum version when $a \rightarrow 0$. The functions $B$ and $C_{\mu}$ are dimensionless, the only dimensionful parameters are $\Lambda$ and the lattice spacing. Note also that the link variables may be introduced 
as part of the 1-body Hamiltonian in Fourier space, just by computing the Fourier transform

$$
\begin{aligned}
\tilde{H}_{ \pm}(n \mid \varphi) & =\sum_{t=1}^{N} H_{ \pm}(a t) e^{-2 \pi i a \varphi \cdot t} e^{-2 \pi i a t \cdot n} \\
& =\sum_{t=1}^{N} H_{ \pm}(a t) e^{-2 \pi i a t \cdot(n+\varphi)}=\tilde{H}_{ \pm}(n+\varphi)
\end{aligned}
$$

which means that the lattice versions of relations (13) hold. In terms of the eigenspinors of $\tilde{H}_{ \pm}(n)$ :

$$
\tilde{H}_{ \pm}(n) u_{ \pm}(n)=\omega_{ \pm}(n) u_{ \pm}(n) \quad \tilde{H}_{ \pm}(n) v_{ \pm}(n)=-\omega_{ \pm}(n) v_{ \pm}(n)
$$

where (see [13])

$$
\begin{aligned}
\omega_{ \pm}(n) & =\frac{1}{a} \sqrt{C^{2}(a n)+(B(a n) \pm a|\Lambda|)^{2}} \\
u_{ \pm}(n) & =\frac{\omega_{ \pm}(n)+B(a n) \pm a|\Lambda|-i \not(a n)}{\sqrt{2 \omega_{ \pm}(a n)\left(\omega_{ \pm}(a n)+B(a n) \pm a|\Lambda|\right)}} \chi \\
v_{ \pm}(n) & =\frac{\omega_{ \pm}(n)-B(a n) \mp a|\Lambda|+i \not(a n)}{\sqrt{2 \omega_{ \pm}(a n)\left(\omega_{ \pm}(a n)-B(a n) \mp a|\Lambda|\right)}} \chi
\end{aligned}
$$

we define the field expansions

$$
\Psi(r)=\frac{1}{N^{2}} \sum_{-\frac{N}{2}}^{+\frac{N}{2}}\left[b_{ \pm}(n \mid \varphi) u_{ \pm}(n \mid \varphi)+d_{ \pm}^{\dagger}(n \mid \varphi) v_{ \pm}(n \mid \varphi)\right] e^{2 \pi i a n \cdot r}
$$

Now the relevant anticommutation relations read

$$
\begin{aligned}
\left\{\Psi(r), \Psi\left(r^{\prime}\right)\right\} & =0 \\
\left\{\Psi(r), \Psi^{\dagger}\left(r^{\prime}\right)\right\} & =\delta\left(r-r^{\prime}\right) \\
\left\{b_{ \pm}(n \mid \varphi), b_{ \pm}^{\dagger}\left(n^{\prime} \mid \varphi\right)\right\} & =\delta_{2 \pi}\left(n-n^{\prime}\right) \\
\left\{d_{ \pm}(n \mid \varphi), d_{ \pm}^{\dagger}\left(n^{\prime} \mid \varphi\right)\right\} & =\delta_{2 \pi}\left(n-n^{\prime}\right)
\end{aligned}
$$

where the periods of the $\delta$-functions are indicated in their corresponding suffixes. The outcome of evaluating the overlap with this conventions, and its $a \rightarrow 0$ limit are discussed in the next two subsections, which deal with the real and imaginary parts of the effective action separatedly. 


\subsection{Real part of $\Gamma$.}

A straightforward calculation along the lines of the corresponding continuum object yields

$$
\operatorname{Re} \Gamma(\varphi, a)=-\sum_{-\frac{N}{2}}^{+\frac{N}{2}} \log \frac{v_{+}^{\dagger}(n+\varphi, a) v_{-}(n+\varphi, a)}{v_{+}^{\dagger}(n, a) v_{-}(n, a)}
$$

where now we only display the dependence of $\Gamma$ on $\varphi$ and the lattice spacing $a$, omitting the $\Lambda$-dependence since this parameter will not be affected by the following treatment. Using the explicit form of the lattice eigenspinors, we can rewrite (66) as 13

$$
\operatorname{Re} \Gamma(\varphi, a)=-\sum_{-\frac{N}{2}}^{+\frac{N}{2}}\{\log \cos \beta(n+\varphi)-\log \cos \beta(n)\}
$$

where

$$
\begin{aligned}
\cos \beta(n) & =\sqrt{\frac{\omega_{+}(n)-a^{-1} B(n)-|\Lambda| \frac{\omega_{-}(n)-a^{-1} B(n)+|\Lambda|}{2 \omega_{+}(n)}}{2 \omega_{-}(n)}} \\
& +\sqrt{\frac{\omega_{+}(n)+a^{-1} B(n)+|\Lambda| \omega_{-}(n)+a^{-1} B(n)-|\Lambda|}{2 \omega_{+}(n)}} .
\end{aligned}
$$

Note that the factor $a^{-1}$ in front of $B$ is necessary to match dimensions $(\Lambda$ is a mass).

We need to check that if we perform the summation over $n_{\mu}$ in (67) and then take the limit $N \rightarrow \infty$, we recover the continuum expression (22) for $\operatorname{Re} \Gamma(\varphi, \Lambda)$. To this end first we note that the dominant contributions to (67) come from the zeroes of $\cos \beta(n)$ and $\cos \beta(n+\varphi)$. This happens when $C_{\mu}$ and $B$ vanish simultaneously. But if $r^{2}>>\Lambda^{2}$, there will be no contribution from the doublers, and the only zeroes of $\cos \beta$ will be at $a n_{\mu} \rightarrow 0$. Hence we can expand (67) in powers of $a$. The leading, i.e., $a=0$ term is convergent and coincides with the continuum limit, since putting $a=0$ everywhere in (67) amounts to replacing both $\omega_{+}$and $\omega_{-}$by their common continuum counterpart $\omega(n)$, and $B$ can be put to 0 in (68), since its small- $a$ expansion begins with $a^{2}$. With these replacements, the function which is summed in 
(67) becomes identical to the one of the continuum overlap calculation and moreover $N=a^{-1}$ tends to infinity, so that the finite sum becomes a series.

One can show that the subleading terms in $a$ are given by convergent series and hence make a vanishing contribution when $a \rightarrow 0$. To see this we first note that (67) is an even function of $a$. Therefore, the first subleading contribution will come from the coefficient of $a^{2}$ in the expansion of (67) in powers of $a$.

The order $a^{2}$ term in the expansion of the function summed in (67) in powers of $a$ turns out, for large $n$, to behave like

$$
\begin{aligned}
\mathcal{O}\left(a^{2}\right) & =a^{2} \frac{1}{2} \Lambda^{2}\left[2 r^{2}\left(\frac{(n+\varphi)^{4}}{\omega^{4}(n+\varphi)}-\frac{n^{4}}{\omega^{4}(n)}\right)+\frac{n_{\mu}\left(n^{\mu}\right)^{3}+\left(n_{\mu}\right)^{3} n^{\mu}}{n^{2} \omega^{2}(n)}\right. \\
& \left.-\frac{(n+\varphi)_{\mu}\left((n+\varphi)^{\mu}\right)^{3}+\left((n+\varphi)^{3}\right)_{\mu}(n+\varphi)^{\mu}}{(n+\varphi)^{2} \omega^{2}(n+\varphi)}\right]
\end{aligned}
$$

Then by expanding (69) in powers of $\varphi$, the order 0 term in $\varphi$ is zero, since it is killed by the normalization we use for the determinant. The term linear in $\varphi$ vanishes when summed over $n$, as can be seen also from the fact that (67) is invariant under $\varphi \rightarrow-\varphi$. But then the term quadratic in $\varphi$ carries a behaviour $n^{-2}$ for large $n$, and thus the sum over $n$ is at worst logarithmically divergent with $1 / a$. When $a$ tends to zero this logarithmic factor is killed by the $a^{2}$ power multiplying this term. Other powers of $\varphi$ tend also to zero, since the term of order $q$ in $\varphi$ carries a $1 / n^{q}$ large- $n$ behaviour.

\subsection{Imaginary part of $\Gamma$.}

Using the explicit form of the lattice eigenspinors, we can write the imaginary part of the effective action for finite lattice spacing as follows

$$
\operatorname{Im} \Gamma(\varphi, \Lambda, a)=\operatorname{Im} \sum_{-\frac{N}{2}}^{+\frac{N}{2}}[\log G(n, \varphi, \Lambda, a)-\log G(n, \varphi,-\Lambda, a)] .
$$

where 


$$
\begin{aligned}
& G(n, \varphi, \Lambda, a)= \\
& {\left[\omega_{+}(n)+\Lambda-a^{-1} B(n)\right]\left[\omega_{+}(n+\varphi)+\Lambda-a^{-1} B(n+\varphi)\right]} \\
& +a^{-2} C(2 \pi a n) \cdot C(2 \pi a(n+\varphi))+\frac{i \epsilon^{\mu \nu}}{a^{2} \tau_{2}} C_{\mu}(2 \pi a n) C_{\nu}(2 \pi a(n+\varphi))
\end{aligned}
$$

It is immediate to realize that, again for the imaginary part, the limit $a \rightarrow$ 0 yields the continuum overlap definition which was already evaluated. It is only necessary to realize that both $\omega_{+}$and $\omega_{-}$tend to their common continuum limit, and that the contributions from $B$ are suppressed by an extra power of $a$. So let us discuss the subleading terms. For any $a \neq 0$, one sees that

$$
G(n, \varphi,-\Lambda, a)=G(n, \varphi, \Lambda,-a),
$$

therefore for $a \neq 0(\sqrt{72})$ can be used in (71) and (70) to deduce:

$$
\operatorname{Im} \Gamma(\varphi, \Lambda,-a)=-\operatorname{Im} \Gamma(\varphi, \Lambda, a),
$$

thus only odd powers of $a$ (except for the $a=0$-term) may appear in a small$a$ series. The first subleading term might then be of order $a$. A calculation of this order- $a$ term for (71) yields a function with the structure

$$
\mathcal{O}(a)=a \sum_{n_{1}, n_{2}} f(n, \Lambda, n+\varphi) \epsilon^{\mu \nu} n_{\mu} \varphi_{\nu}
$$

where $f$ is given by:

$$
\begin{aligned}
f(n, \Lambda, n+\varphi)=\frac{r}{\tau_{2}}\left(\frac{n^{2}}{\omega(n)}+\frac{(n+\varphi)^{2}}{\omega(n+\varphi)}\right) \times \\
{\left[\frac{(\omega(n+\varphi)+\Lambda)(\omega(n)+\Lambda)}{[(\omega(n+\varphi)+\Lambda)(\omega(n)+\Lambda)+n \cdot(n+\varphi)]^{2}+n^{2} \varphi^{2}-(n \cdot \varphi)^{2}}\right.} \\
\left.-\frac{(\omega(n+\varphi)-\Lambda)(\omega(n)-\Lambda)}{[(\omega(n+\varphi)-\Lambda)(\omega(n)-\Lambda)+n \cdot(n+\varphi)]^{2}+n^{2} \varphi^{2}-(n \cdot \varphi)^{2}}\right] .
\end{aligned}
$$

Equation (75) is quite a cumbersome scalar function of its arguments, but we only need to know that it behaves as $1 / n$ for large $n$, and it depends on $\varphi$ only through the combination $n+\varphi$. Thus the series (74) appears to be quadratically divergent. Note that the imaginary part of (71) is an even function of $\varphi_{\mu}$, so, even though the terms of order two and higher in an expansion of $f$ in powers of $\varphi$ will tend to zero as for the real part case, we 
are still left with the term of order one. This will however vanish, because expanding $f$ to first order in $\varphi$ will yield for (74)

$$
\mathcal{O}(a) \sim a \sum_{n_{1}, n_{2}} h(n, \Lambda) \epsilon^{\mu \nu} n_{\mu} \varphi_{\nu} n \cdot \varphi
$$

where $h$ is also a scalar function but of $n$ and $\Lambda$, whose form is not relevant to our argument. Since one can prove that

$$
\sum_{n_{1}, n_{2}} h(n, \Lambda) n^{\mu} n^{\nu} \propto g^{\mu \nu}
$$

where $g$ is the metric tensor. Inserting (77) into (76) yields 0 for this term, since $\epsilon^{\mu \nu} \varphi_{\mu} \varphi_{\nu}=0$.

This completes the proof of the vanishing of the subleading term for the imaginary part.

\section{Acknowledgements.}

I am grateful to S. Randjbar-Daemi for many useful discussions and suggestions, and to H. Neuberger and R. Narayanan for reading the manuscript, and useful correspondence.

\section{References}

[1] R. Narayanan and H. Neuberger, Nucl. Phys. B443:305-385 (1995). See also: R. Narayanan, H. Neuberger and P. Vranas, hep-lat/9509046; R. Narayanan and H. Neuberger, hep-lat/9509047; H. Neuberger, hep-lat/9511001.

[2] D. B. Kaplan, Phys. Lett. B288 342 (1992).

For a recent review of Kaplan's formulation, see: K. Jansen, hep-lat/9410018.

[3] L. H. Karsten, Phys. Lett. 104B, 315 (1981);

L. H. Karsten and J. Smit, Nucl. Phys B183, 103 (1981);

H. B. Nielsen and M. Ninomiya, Nucl. Phys B185, 20 (1981); Nucl. Phys B193, 173 (1981); Phys. Lett. 105B, 219 (1981). For a recent review see: Y. Shamir, hep-lat/9509023. 
[4] R. Narayanan and H. Neuberger, Nucl. Phys. B 412, 574 (1994).

[5] S. Randjbar-Daemi and J. Strathdee, Phys. Rev. D51:6617-6619 (1995)

S. Randjbar-Daemi and J. Strathdee, Phys. Lett. B348:543-548 (1995).

[6] S. Randjbar-Daemi and J. Strathdee, ICTP preprint IC-95-305, hep-th/9510067.

[7] T. Kawano and Y. Kikukawa, hep-lat/9501032.

[8] D.B. Kaplan and M. Schmaltz, hep-th/9510197.

[9] S. Aoki and R. Levien, Phys. Rev. D 51:3790-3802 (1995).

[10] R. Narayanan and H. Neuberger, Phys. Lett. B348:549-552 (1995).

[11] C.D. Fosco and S. Randjbar-Daemi, Phys. Lett. B354:383-388 (1995).

[12] L. Alvarez-Gaume, G. Moore and C. Vafa, Comm. Math. Phys. 106 1, (1986).

[13] S. Randjbar-Daemi and J. Strathdee, Nucl. Phys. B443:386-416 (1995).

[14] R. Narayanan, H. Neuberger and P. Vranas, hep-lat/9503013 